\title{
Saúde e Sustentabilidade: desafios conceituais e alternativas metodológicas paraa análise desistemas locais de Segurança Alimentar e Nutricional.
}

\section{Health and Sustainability: conceptual challenges and alternatives methodology for the analysis of analysis of local systems of Food and Nutritional Security.}

\section{Salud y Sostenibilidad: retos alternativas conceptuales y metodológicos para el análisis de los sistemas locales de seguridad alimentaria y nutrición.}

Luciene BURLANDY ${ }^{1}$

Renato MALUF

Rosana MAGALHÃES ${ }^{3}$

Márcio REIS ${ }^{4}$

Luiz MAFRA 5

Daniela FROZI

RESUMO: Processos de desenvolvimento sustentáveis envolvem estratégias cooperativas, diálogo interdisciplinar e articulação intersetorial. O foco exclusivo em setores ou aspectos isolados da dinâmica social dificilmente será capaz de apoiar a conformação de sistemas de governança capazes de enfrentar os novos desafios. Buscando analisar experiências e resgatar aprendizados em torno da implementação de ações públicas intersetoriais, este trabalho apresenta os pressupostos metodológicos do enfoque sistêmico na análise da implementação de Sistemas Locais de Segurança Alimentar e Nutricional (SAN) e sua relação com o debate sobre modelos de desenvolvimento sustentável. Na definição desses pressupostos, leva-se em consideração o contexto (bioma, densidade sócio-organizacional, reprodução socioeconômica das famílias e institucionalidade), as condições de SAN das famílias e o sistema alimentar, tendo em conta quatro dimensões de análise: intersetorialidade, descentralização, participação e equidade. São exploradas as interfaces e correlações existentes entre a promoção de sistemas descentralizados de SAN e modelos sustentáveis de desenvolvimento. Por último, é proposto um modelo de referência para o

1 Professora Associada da Faculdade de Nutrição da Universidade Federal Fluminense. Rua Mario Santos Braga, 30, $4^{\circ}$ andar, Centro. CEP: 24020-140. Niterói, RJ.

E-mail: burlandy@uol.com.br

2 CPDA, Universidade Federal Rural do Rio de Janeiro

3 Fundação Oswaldo Cruz. Escola Nacional de Saúde Pública

4 CERESAN, Universidade Federal do Rio de Janeiro e FEAD-MINAS

5 Instituto de Ciências Sociais Aplicadas, Universidade Federal de Alfenas

6 Fundação Oswaldo Cruz, DIREB, PALIM, DF 
acompanhamento de processos de tomada de decisão e conformação de desenhos organizacionais capazes de promover diálogo e contornar conflitos de interesses.

Palavras Chave: Sustentabilidade, Segurança Alimentar e Nutricional e Desenvolvimento.

\begin{abstract}
Sustainable development processes involve cooperative strategies, interdisciplinary dialogue and intersectoral coordination. The exclusive focus on individual sectors or aspects of social dynamics is unlikely to be able to support the formation of systems of governance capable of facing new challenges. Seeking to analyze experiences and learning rescue around the implementation of intersectoral public's actions, this paper presents the methodological assumptions of the systems approach in analyzing the implementation of Local Systems of Food Security and its relation to the debate on sustainable development models. In defining these assumptions, it takes into account the context (biome, density, socio-organizational, socioeconomic reproduction of families and institutions), the conditions of Food Security families and the food system, taking into account four dimensions of analysis: intersectionality, decentralization, participation and equity. It explores the interfaces and relationships between the promotion of decentralized Food Security Systems and sustainable models of development. Finally, we propose a reference model for the monitoring of processes of decision making and shaping of organizational designs that promote dialogue and solving conflicts of interest.
\end{abstract}

Keywords: Sustainability, Food Security and Development.

RESUMEN: Procesos de desarrollo sostenible implican estrategias de cooperación, diálogo interdisciplinario y la coordinación interinstitucional. El enfoque exclusivo en sectores o aspectos aislados de la dinámica social no será capaz de apoyar la formación de los sistemas de gobierno para contestar a los nuevos desafíos. Tratando de analizar experiencias y aprender sobre el rescate alrededor de la implementación de las acciones públicas intersectoriales, este trabajo presenta los supuestos metodológicos del enfoque sistémico en el examen de la aplicación de los sistemas de seguridad alimentaria local y nutricional (SAN) y su relación con el debate sobre los modelos de desarrollo sostenible. Al establecer estos supuestos, se tiene en cuenta el contexto (bioma, la densidad social y organizacional, la reproducción socioeconómica de las familias e instituciones), condiciones de SAN de las familias y el sistema de alimentos, teniendo en cuenta cuatro dimensiones de análisis: interseccionalidad, decentralization, la participación y la equidad. Interfaces y correlaciones entre la promoción de la descentralización SAN y sistemas modelo de desarrollo sostenible se exploran. Por último, se propone un modelo de referencia para los diseños organizativos de toma de decisiones y dar forma a los procesos de seguimiento que promuevan el diálogo y superar los conflictos de intereses.

Palabras llave: sostenibilidad, seguridad alimentaria y nutrición y desarrollo. 


\section{INTRODUÇÃO}

Às vésperas da Conferência Rio + 20, ainda existiam ambivalências e dúvidas acerca dos avanços no processo de formulação de políticas públicas sustentáveis. Frente ao desafio da construção de alternativas sustentáveis de consumo e produção, fez surgir a necessidade de maior reflexão e debate. Um dos obstáculos para a sustentabilidade dos processos de desenvolvimento em diferentes territorialidades está na conformação de sistemas de governança capazes de promover e objetivar o diálogo entre atores com perspectivas e interesses distintos. E, neste contexto, uma questão que surge é a definição de temas e enfoques que, ao mesmo tempo, promovam consensos e estimulem a implementação de iniciativas mais robustas de desenvolvimento social.

Especialmente no campo da segurança alimentar e nutricional (SAN), políticas, programas e ações vêm sendo implementados em diferentes países. Embora as concepções em torno do conceito de SAN possam variar, diferentes estudos avaliativos têm revelado aspectos diversos das estratégias adotadas em distintos contextos. Este processo vem contribuindo para a consolidação da SAN também como um campo de produção de conhecimento, marcado pela diversidade de enfoques analíticos.

No caso brasileiro as duas últimas décadas evidenciaram o processo fértil de construção de novas abordagens sobre SAN, compartilhado por organizações sociais, setores de governo e instituição acadêmicas $\left({ }^{1}\right)$. O debate avançou na formulação do conceito amplo de SAN, qual seja: a realização do direito humano à uma alimentação saudável, acessivivel, de qualidade, em quantidade suficiente e de modo permanente, sem comprometer o acesso a outras necessidades essenciais, com base em práticas alimentares saudáveis, respeitando as diversidades culturais, e realizando-se em bases sustentáveis do ponto de vista sócio-econômico e agro-ecológico ().

A amplitude e complexidade do conceito de SAN associados à ótica do Direito Humano à Alimentação Adequada e Soberania Alimentar pautaram o debate sobre as dimensões e fluxos prioritários do Sistema Nacional de SAN (SISAN) formalizado em 2006. As ações de diferentes setores de governo e sociedade no âmbito da produção, abastecimento, comercialização e consumo de alimentos orientadas pelo conceito amplo de SAN trouxeram, desta forma, possibilidades, mas também desafios para o espectro de intervenções na área. Além disso, o conceito de SAN também suscitou intensos debates metodológicos em torno da sistematização de experiências locais.

Diferentes estudos propuseram-se a desenvolver instrumentos de avaliação que abarcam distintos aspectos desse processo, tais como: a construção da agenda nacional em torno do tema e a formulação da Lei Orgânica ${ }^{3}$, o impacto da política ${ }^{4}$, a implementação local de um conjunto de programas ${ }^{5}$, a implementação local de programas específicos que são considerados como parte da política ${ }^{6}$; a implementação local de Sistemas de SAN 3.

O presente artigo apresenta o marco analítico adotado em pesquisa realizada pelos autores que 
teve como objetivo analisar potencialidades e limites para implementação de Sistemas Locais de SAN (SistlocSAN) em três municípios de pequeno porte, situados em regiões de significativa vulnerabilidade sócio-econômica dos estados de Minas Gerais, Bahia e Rio de Janeiro. O estudo articulou as condições de saúde individual e coletiva às dimensões econômico-produtiva e políticoinstitucional e favoreceu a compreensão sobre o envolvimento dos diferentes atores, os processos de pactuação e as possíveis repercussões das políticas e iniciativas públicas.

O desafio principal foi avançar em estratégias metodológicas e marcos analíticos capazes de apoiar a apreensão da dinâmica complexa de aspectos produtivos, de consumo, nutricional e institucional em cada realidade. ${ }^{7}$

São apresentados a base teórica e conceitual utilizada no estudo e sua operacionalização em instrumentos e métodos de coleta de dados, propondo por fim uma matriz de análise de Sistemas Locais de SAN que articulou três enfoques distintos, a saber: a) o enfoque de SAN; b) o enfoque sistêmico; c) o enfoque de desenvolvimento local. A escolha destes enfoques foi pautada pela própria natureza complexa e inovadora do objeto de pesquisa, na medida em que o foco da investigação foi a análise das condições de implementação de SisLocSan, entendidos como "unidades imbricadas", experiências que evidenciam interdependências e diferentes perfis de interação entre múltiplas variáveis. Assim, a abordagem sistêmica que "sugere a associação de diferentes lógicas de análise" ${ }^{8}$, juntamente com as referências conceituais de SAN e desenvolvimento local, tornaram-se os principais eixos teóricos utilizados para explicar os limites e as potencialidades dos processos de implementação de SisLocSan. O presente artigo irá se debruçar sobre as interelações entre os dois primeiros enfoques (de SAN e Sistêmico).

\section{O processo de construção da abordagem analítica do estudo}

Ao considerar os dois enfoques adotados e, ao mesmo tempo, confrontar o conceito de SAN 2 com a dinâmica das experiências locais, o primeiro passo foi discriminar quais ações e intervenções seriam consideradas em cada contexto. Assim, reconhecendo que os principais circuitos de SAN encontram-se nas esferas da produção, distribuição, comercialização, acesso, consumo e utilização biológica de alimentos, foi importante eleger uma via capaz de favorecer um processo sistemático e articulado de aproximação da realidade. A esfera do acesso aos alimentos, no caso, possibilitou não só avançar na abordagem das múltiplas interações entre as dimensões da SAN, mas também na interface com o debate sobre equidade. Frente à complexidade dos múltiplos critérios ligados ao conceito de SAN, o caminho metodológico adotado buscou contornar os limites de uma investigação de corte transversal. Ao mesmo tempo, privilegiou o princípio da equidade, caro na discussão contemporânea sobre as políticas sociais e de saúde. ${ }^{9}$

No que se refere às dimensões de análise, o estudo pressupôs que essas fossem referenciadas pelas diretrizes e princípios norteadores do Sistema de SAN, que já estavam indicados em Conferências Nacionais e foram posteriormente institucionalizados na Lei Orgânica. Como indicam os estudos 


\section{$59 / /$}

sobre outros sistemas, o desenho metodológico depende da clareza sobre os princípios, objetivos e metas estabelecidas e que vão embasar a escolha das dimensões que serão objeto de análise ${ }^{10}$. No caso do SISAN, as diretrizes referem-se à promoção da intersetorialidade, descentralização, monitoramento, conjugação de ações imediatas e estruturais e articulação entre orçamento e gestão. Os princípios que o regem são: a universalidade, equidade, participação social e transparência das intervenções, incluindo os critérios seletivos de usuários, 2.

\section{Operacionalização analítica dos princípios e conceitos}

Os princípios e diretrizes do SISAN e os respectivos conceitos aqui apresentados foram operacionalizados no estudo através dos seguintes e respectivos componentes:

a) Intersetorialidade. A análise privilegiou os espaços e mecanismos institucionais de aproximação entre setores; a incorporação ou aproximação das diferentes dimensões e abrangência da SAN; os mecanismos de distribuição dos recursos entre os setores; a possível existência de pólo(s) aglutinador(es) do tema da SAN na instância respectiva; as alianças entre segmentos de determinados setores do governo (exemplo: responsáveis pela compra da alimentação escolar e gestores da agricultura) que dificultam ou facilitam ações integradoras; concepção dos atores locais sobre integração entre programas. Foram considerados marcadores potenciais de intersetorialidade: a existência de ações conjuntas desenvolvidas por diferentes setores de governo. Num nível mais avançado, estas ações articulam produção e consumo de alimentos; existência de espaços institucionais de pactuação entre setores; existência de espaços de articulação intersetorial cotidiana, como grupos de trabalho;

b) Descentralização. Foi dada relevância ao estudo das formas de incentivo; aos mecanismos utilizados para estabelecer papéis e atribuições das esferas de governo; as arenas e fluxos de integração intergovernamental e com as organizações sociais; aos incentivos dos níveis subnacionais de governo para adesão dos demais: apoio técnico - financeiro. Foram considerados como marcadores potenciais de descentralização: existência de mecanismos de monitoramento local da utilização dos recursos e do processo de implementação; existência de canais institucionais de diálogo entre os níveis de governo; grau de participação do governo estadual na implementação dos programas; iniciativas de formação de gestores locais pelos demais níveis de governo; conexões a partir dos circuitos econômicos em torno da cadeia alimentar entre o governo local, o estadual e o federal.

b) Equidade. Analisou-se as desigualdades de acesso à alimentação adequada; os princípios de equidade na formulação e implementação de ações; os processos sociais mais amplos que geram desigualdades; formas de interação entre os atores sociais que evitem o reforço de desigualdades; a adequação e a transparência dos critérios de elegibilidade e seletividade das ações e intervenções propostas. Foram considerados marcadores potenciais de equidade: processos de seleção dos usuários dos programas com ampla divulgação pública ou captação domiciliar; transparência dos critérios seletivos dos programas e ações; participação das organizações sociais; identificação dos 
segmentos dos produtores locais que conseguem assumir espaços públicos e de que forma isto facilita seu acesso aos recursos existentes; existência de programas voltados para os segmentos mais vulneráveis; programas de inserção dos grupos mais vulneráveis em processos de agregação de valor aos produtos, no acesso ao crédito, na garantia do escoamento dos produtos e em processos de autonomização financeira.

c) Participação social. Foram descriminados e analisados os espaços e mecanismos de participação na formulação, implementação e monitoramento, tais como existência de instâncias de participação; a natureza dos fluxos de informação; os mecanismos de responsabilização dos gestores e demais atores sociais; a participação nas diferentes fases da cadeia alimentar. Foram considerados marcadores potenciais de participação social: existência de mecanismos públicos de denúncia em caso de violação; existência de mecanismos públicos de responsabilização dos gestores; existência de espaços institucionais de participação no processo de implementação dos programas; decisões dos espaços de controle social que redundam em ações concretas; grau de conhecimento dos conselheiros sobre os programas; existência de formas sistemáticas de divulgação sobre o desenho operacional e etapas da implementação do programa; existência de iniciativas de mobilização do crédito (como fator produtivo) através das cooperativas.

\section{O enfoque sistêmico}

A utilização da perspectiva sistêmica para abordar a SAN é bastante recente no Brasil, bem como seu desenvolvimento conceitual. A instituição formal de um Sistema Nacional de SAN (SISAN) foi referenciada pela Lei Orgânica de SAN 2.

Como dito, no que se refere ao marco analítico, a apropriação da perspectiva sistêmica focou-se tanto na organização das ações e instituições (Sistema Institucional) quanto dos fluxos e processos locais de produção, comercialização e consumo. (Sistema Alimentar). O estudo partiu da premissa que a realidade tem natureza sistêmica a ser apreendida com o instrumental analítico fornecido pelas teorias sobre sistemas. Ao mesmo tempo, a identificação dos elementos que compõem um sistema determinado permite potencializar os ganhos sinérgicos gerados pelas relações sistêmicas entre tais elementos por intermédio da institucionalização e aprofundamento dessas relações.

Um dos precursores do chamado pensamento sistêmico, Bertalanffy define a noção de sistema como "um complexo de elementos em interação" ambientais e de ecossistemas, cuja compreensão imediata, mesmo que comportando imprecisões, advém da referência às relações funcionais existentes entre os elementos que compõem a natureza e à interação do ser humano com esses elementos. Alguns critérios do pensamento sistêmico, segundo Capra ${ }^{12}$, podem ser assim sistematizados: o mais geral é a mudança das partes para o todo; as propriedades das partes surgem das "relações de organização" entre elas, isto é, as partes são apenas um padrão numa teia inseparável de relações; a capacidade de deslocar a própria atenção de um lado para outro entre níveis sistêmicos; ser um pensamento não analítico, mas sim contextual; 
a percepção do mundo vivo como uma rede de relações; a estrutura vista como manifestação de processos subjacentes, tornando o pensamento sistêmico sempre como um pensamento processual.

Sistemas sociais, sistemas econômicos e sistemas políticos, entre outras, são formulações de uso igualmente difundido, com distintas conceituações e aplicações e, por certo, algumas controvérsias acerca da extensão de sua aplicabilidade em processos que, no mais das vezes, comportam elevado grau de incerteza e de abertura de soluções. Não obstante, autores como Santos ${ }^{13}$ identificam avanços significativos pela aplicação da abordagem sistêmica nas Ciências Sociais, por contribuírem para o estabelecimento de uma visão do mundo "não dualista", apesar de achar ainda modestas suas consequências práticas.

A perspectiva sistêmica aqui adotada filia-se aos enfoques que caracterizam o sistema como um conjunto composto de interrelações entre seus elementos que evolui com contradições, isto é, as relações sistêmicas presentes nas dinâmicas econômicas, sociais e políticas contêm elementos de conflito. Supõe, ademais, que a ação humana se faz num ambiente de incerteza (desconhecimento em relação ao futuro) e recorre ao conceito de 'consequências não intencionais' da ação humana com o sentido de abertura a várias soluções. Portanto, admite a possibilidade das soluções abertas, seguindo autores como Hirschman e Sen. Essa premissa é radicalmente distinta das soluções fechadas características, por exemplo, dos usos formalizados do enfoque sistêmico em modelos que pretendem descrever complexas realidades econômicas e sociais na forma de sistemas de equações ${ }^{7}$.

Este entendimento permite-nos recorrer, complementarmente, a outro instrumento analítico que também explica as inter-relações entre as atividades resultando num conjunto social não harmônico. Trata-se da noção desenvolvida por Hirschman ${ }^{14}$ de encadeamentos "para frente" e "para trás" (forward and backward linkages), ampliados para além dos elos entre atividades econômicas. Ela incorpora a variável tempo e conclui por uma dinâmica desequilibrada quando se coloca em marcha de mecanismos indutores, sobretudo, indutores de decisões pelos agentes presentes nas várias etapas das cadeias assim formadas. Antes que um problema, a geração de desequilíbrios é tida como dinamizadora das estruturas sociais que aqueles efeitos e mecanismos contribuem, permanentemente, para redefinir - "desenvolvimento é promover desequilíbrios". Dinâmicas sistêmicas portadoras de contradições e mecanismos indutores causadores de desequilíbrio constituem duas referências analíticas apropriadas para abordar nosso objeto, que é a promoção de SisLocSan articulada a estratégias de desenvolvimento.

Uma outra referência preliminar, relacionada com as anteriores, é a importância atribuída à experiência, ou melhor, ao aprendizado individual e coletivo nos comportamentos que conformam as trajetórias dos atores sociais e as ações públicas por eles promovidas. Ela nos leva a evitar o risco dos determinismos e também os conhecidos excessos no campo das pretensões prescritivas,

7 Ela não coincide, também, com a caracterização dos sistemas abertos feita por Capra (2003) na qual a auto-regulação é a propriedade chave e, embora aberto ao mundo exterior e não contendo sempre os componentes idênticos, é um sistema aberto num estado (quase) estacionário. 
sem abdicar, inteiramente, da perspectiva normativa inerente à abordagem de estratégias de desenvolvimento e ao próprio objetivo da $\mathrm{SAN}^{15}$.

O papel do aprendizado e o suposto das 'soluções abertas' integram uma opção metodológica, sustentada por Hirschman 14, pelo estilo cognitivo - em lugar do pensar paradigmático - que explora as possibilidades de atuação frequentemente em forma dissonante, desequilibradora e com 'sequencias invertidas'. Mesmo o maior rigor formal das análises de Amartya Sen não o impede de admitir o desenvolvimento como um conceito incompleto de forma permanente no que se refere ao ordenamento dos seus fins, dadas as avaliações divergentes quanto ao que se considera valioso promover ou ao que seria uma vida valiosa ${ }^{16}$.

A abordagem sistêmica da SAN adotada no estudo parte das premissas acima descritas sobre dinâmicas sistêmicas conflitivas e desequilibradas, soluções abertas e papel do aprendizado. Como notado em Santoro e Pinheiro ${ }^{17}$, Josué de Castro já apontava, no seu 'Geografia da Fome' (1946), que um dos grandes problemas para o planejamento de soluções para a fome é a própria abordagem do planejamento que não considera o problema de forma conjunta e sistêmica, como um complexo de manifestações simultaneamente biológicas, econômicas e sociais. Nessa direção, e acrescentando o fator tempo, Barraclough ${ }^{18}$ afirma que a pobreza massiva e a fome têm natureza sistêmica sendo possível, no curto prazo, obter melhorias em alguns aspectos da segurança alimentar independentemente de outros; no longo prazo, porém, todas as dimensões devem avançar juntas, pois do contrário "o sistema alimentar se tornará crescentemente instável” (grifo nosso).

Pode-se, contudo, antecipar a utilidade do instrumental analítico fornecido pelo enfoque sistêmico para apreender muitas das questões envolvidas pela SAN. Importa observar, nos assentamentos humanos, a complexidade de interações e relações que neles acontecem e como se dá o fluxo energético necessário para sua manutenção e estruturação. No caso, quais os circuitos pelos quais caminham o alimento (e a água), considerando-se a produção, a distribuição e acesso, o consumo e sua utilização biológica, presentes nas localidades.

Reconhecer e evidenciar tais fluxos e circuitos é fundamental, primeiramente, porque é aqui que se propicia grande parte da sobrevivência material de uma sociedade e também porque, dentre os inúmeros pontos que constituem uma cultura, a sobrevivência material é um elo forte e central na constituição da teia da subjetividade de uma localidade. O enfoque sistêmico que considere as complexidades biológicas, econômicas e sociais, torna mais evidente os papéis da questão alimentar e nutricional dentro dos processos humanos e, deste modo, traz também à tona a importância do tema alimentar como detonador de dinâmicas transformadoras. Em síntese: os sistemas só podem ser percebidos de maneira integrada, interna e externamente e há sempre um princípio de coordenação que possibilita essa integração; os sistemas se mantêm, se reproduzem e se transformam.

Assim, tendo em vista os objetivos que nortearam a presente pesquisa, serão colocados em 
evidência os elementos do enfoque sistêmico que contribuiriam para a formulação, implementação e monitoramento de ações e políticas públicas de SAN de um modo que configuraria os pretendidos SisLocSAN.

Para caracterizar um sistema, é preciso identificar a presença de, pelo menos, dois elementos, a saber, fluxos de interdependência e mecanismos de coordenação entre os componentes do sistema em questão. Dois motivos principais justificam a adoção dessa abordagem, uma razão de escala e outra ligada ao fenômeno dos encadeamentos. A questão alimentar se manifesta em distintas escalas - desde os indivíduos e domicílios até os âmbitos nacional e global - as quais interagem entre si em diversos aspectos, dando origem a relações de condicionamento e de determinação entre elas. Por exemplo, a condição alimentar dos indivíduos é afetada por fatores de ordem geral que condicionam a evolução do emprego e da renda, enquanto que a condição alimentar da população de um país pode ser alterada como resultado das ações desenvolvidas, localmente, junto aos indivíduos e às famílias.

Já os encadeamentos estabelecidos pelos 'fluxos de interdependência' nos obrigam a considerar os reflexos sistêmicos da ação dos fatores 'exógenos' sobre os componentes 'endógenos' de um dado sistema, bem como as relações simbióticas, as interações e sinergias construídas na ação dos fatores endógenos e exógenos a esse sistema. Por exemplo, os impactos de uma ação 'externa' a um sistema de âmbito local (programas de transferência de renda) não se limitam ao componente diretamente afetado por ela (famílias beneficiadas), pois ele transmite ao menos parte desse impacto para outros componentes (produção e comércio locais); assim como as sinergias geradas pela interação entre os atores locais envolvidos numa dada ação ou programa público (aquisição local de alimentos) podem dar origem a iniciativas que vão além do objetivo primeiro dessa ação ou programa (transferência dos alimentos a uma instituição demandante).

No plano analítico, os fluxos de interdependência integram a base conceitual das análises sobre a questão alimentar, notadamente, na dimensão econômica da produção, comercialização e consumo dos alimentos. Há tempos, consagrou-se a abordagem dos encadeamentos entre as etapas das chamadas cadeias agroalimentares, ou das inter-relações que conformam sistemas alimentares (global, nacionais ou sub-nacionais).

No plano normativo, o próprio enfoque de promoção da SAN incorpora a visão de fluxos de interdependência, na medida em que busca promover a intersetorialidade e a correspondente implementação de ações integradas. Como consequência, em termos operacionais, as ações e programas de SAN dão origem a fluxos de interdependência que vão desde as decisões integradas envolvendo mais de um setor de governo e as organizações sociais, até a operação conjunta dessas ações e programas.

Com relação aos mecanismos de coordenação, sua existência pode ser um produto, por um lado, das relações que se estabelecem entre os agentes econômicos e os atores sociais em geral (espaços 
de interação, associativismo, redes de proteção social, etc.), inclusive, do funcionamento dos mecanismos de mercado. Vários autores demonstraram como os mercados são uma construção social que se dá sob a égide das instituições (normas, convenções e rotinas) que caracterizam uma dada formação social, ao mesmo tempo em que essa construção é parte formadora da institucionalidade. Essa construção pode dar origem a espaços de coordenação mais ou menos formalizados - como é comum ocorrer em várias cadeias agroalimentares e agroindustriais - como também a procedimentos comuns (barganhar, negociar, promover ajustamentos mútuos, etc.). Esses, para Hirschman ${ }^{19}$, expressam os efeitos integrativos dos mercados, não percebidos pelos economistas que, ao pretenderem dotar o sistema de mercado de legitimidade econômica (via modelos de concorrência), frequentemente, sacrificam sua legitimidade sociológica.

Como dito, o espaço institucional que funcionaria como mecanismo principal de coordenação dos Sistemas Institucionais de SAN (SISAN) são os Conselhos de SAN (CONSEA's) nos três níveis de governo (federal, estadual e municipal). Os CONSEA's não são o único componente das dinâmicas de coordenação que propiciam (ou entravam) fluxos sustentáveis dos SISAN's, cabendo verificar essas dinâmicas em cada contexto.

Esses conselhos constituem espaços intersetoriais de diálogo e pactuação entre os vários agentes envolvidos (setores governamentais ou não), com vistas a construir ações integradas e promover a participação social. Os conselhos atuam nos limites da unidade político-administrativa que o define - Município, Estado e União - enquanto que há fluxos de interdependência que extrapolam os limites dessa unidade, cuja incidência gera efeitos sistêmicos no interior dela. A percepção e coordenação dos circuitos/fluxos no nível regional pode enfrentar dificuldades quando circunscritas à unidade administrativa. Entraves podem também ser gerados pela visão dicotômica urbano-rural, da qual deriva a duplicidade de programas, projetos, ações e orçamento, estagnando sinergias entre os elos da cadeia e o planejamento territorial. Esses fatos colocam desafios específicos para o desempenho da função de coordenação atribuída aos Conselhos, quase sempre colocando a necessidade de estabelecer formas de cooperação entre as esferas.

Em princípio, um SISAN se caracteriza como um sistema aberto, em função do seu propósito de 'organizar e monitorar as ações e políticas públicas dos diversos setores governamentais e não-governamentais, articuladas numa política (nacional, estadual ou municipal) de SAN'. Essa opção faz com que esse sistema tenha reduzido grau de autonomia (auto-suficiência) em relação ao contexto em que se insere, diferentemente dos sistemas fechados, que organizam e implementam políticas ou programas específicos, quase sempre setoriais e com fundos orçamentários próprios. Para fins de comparação, o SUS se enquadraria na categoria de sistema fechado, sem com isto desconsiderar que ele interage com outros setores de governo e com as organizações sociais.

Diferentemente dos sistemas setoriais que gerem programas próprios - nesse sentido, são fechados - as políticas de SAN têm a pretensão de sugerir diretrizes e propor ações integradas 
envolvendo participantes de outros sistemas ou processos decisórios, como são os casos do Sistema Único de Saúde, de Assistência e Programas como o de Alimentação Escolar (PNAE) e Apoio a Agricultura Familiar (PRONAF), dentre outros. Assim, envolvem processos decisórios mais complexos, critérios peculiares de representação em seus espaços próprios de coordenação e procedimentos inter-setoriais para implementar e monitorar ações integradas. Pense-se, por exemplo, na integração dos programas de apoio à agricultura familiar e de alimentação escolar, ou entre programas sociais de transferência de renda e de geração de trabalho e renda.

\section{A operacionalização do enfoque Sistêmico}

A concepção de Sistema de SAN adotada na pesquisa refere-se a duas dimensões: 1) a do Sistema Alimentar existente em cada localidade, que diz respeito às dinâmicas concretas, aos agentes e aos fluxos, sociais, políticos e econômicos que atravessam desde a produção até o consumo dos alimentos. 2) O Sistema Institucional, ou seja, o conjunto de instituições, políticas, programas e os atores que afetam a situação de SAN local. Estes dois sistemas interagem e são mutuamente afetados. Portanto, o formato institucional possível em cada localidade é condicionado pela dinâmica de produção e acesso a riquezas, distribuição e concentração destes recursos, incluindo recursos de poder político. Numa via de mão dupla, a própria disponibilidade de serviços e instituições pode afetar a situação de SAN populacional.

Estudos que propõem marcos analíticos para avaliação de sistemas de saúde também partem desta diferenciação entre os sistemas de saúde, que referem-se à situação de saúde e condições de vida de uma dada população e os sistemas de serviços de saúde, que abarca as instituições e ações de prestação de serviços 11 .

Estes Sistemas por sua vez são condicionados por processos que definem um dado contexto local. Desta forma o estudo pressupôs que a inserção das famílias nas dinâmicas sistêmicas que afetam sua condição de SAN se explica ou é influenciada por um conjunto de fatores relacionados às seguintes dimensões dos contextos locais: (a) o contexto biofísico; (b) a reprodução socioeconômica das famílias; (c) a densidade sócio-organizacional; (d) a institucionalidade e o acesso a bens e serviços públicos (Quadro 1).

Assim, as informações coletadas nos municípios objetivaram compreender estas distintas dimensões, bem como os elementos constitutivos do sistema alimentar e do sistema institucional e as possíveis interelações entre eles em cada contexto.

\section{Instrumentos de coleta e análise de dados}

A partir da perspectiva teórica acima descrita, foram construídos os instrumentos e métodos de 
coleta de dados. O desenho geral da pesquisa seguiu os princípios da investigação por triangulação de métodos ${ }^{20},{ }^{21}$ e combinou instrumentos quantitativos e qualitativos complementando-os na análise do tema estudado. Além disto, considerando que a concepção teórica que pautou o estudo exige um profícuo diálogo interdisciplinar, privilegiou-se a composição multidisciplinar da equipe de pesquisadores (incluindo áreas como saúde, economia, sociologia e nutrição) e o complexo diálogo entre diferentes filiações teórico-metodológicas.

Este diálogo foi marcado também pelo intercâmbio interinstitucional, já que a equipe foi composta de integrantes de universidades e organizações Sociais, o que permitiu associar à reflexão conceitual e metodológica aspectos fundamentais ligados ao aprendizado organizacional das diferentes instituições. Esta equipe desenhou de forma conjunta tanto os métodos como os instrumentos de coleta de dados e também foi responsável por coletar os dados dos respectivos estudos de caso e analisá-los.

As informações coletadas em cada município incluíram: 1) dados secundários sobre disponibilidade/produção de alimentos, abastecimento alimentar, acesso aos alimentos, adequação sanitária e nutricional, estado nutricional e perfil de consumo alimentar; dados históricos e socioeconômicos dos municípios no sentido de correlacionar o conjunto de informações obtidas em cada contexto local (2) entrevistas com organizações sociais, gestores públicos, integrantes de conselhos municipais relacionados com a SAN (Conselho Municipal de SAN; de Saúde, Educação, Assistência Social e Desenvolvimento Rural) agentes implementadores de programas específicos que potencialmente afetam desde a produção até o consumo; administradores de restaurantes e estabelecimentos de venda de alimentos (hortifrutis, supermercados). O objetivo destas entrevistas foi mapear os atores, ações e espaços institucionais relevantes, governamentais e não governamentais, além de obter diferentes perspectivas sobre o tema do estudo 20.

Ainda neste sentido, foram também entrevistadas famílias residentes nas áreas urbanas e rurais de cada município, visando compreender suas concepções sobre o sistema produtivo local e as formas de acesso aos alimentos, bem como sua adesão a programas sociais e a estratégias de participação social, além de qualificar as informações de dados secundários.

A definição das famílias entrevistadas não pretendeu atender aos requisitos de construção de uma amostra representativa do ponto de vista quantitativo, que permitiria obter indicadores para o conjunto das famílias do município. A opção foi construir um método qualitativo cujo ponto de partida foi a diferenciação dos bairros ou comunidades urbanas e rurais do município, segundo os fatores que explicam ou influenciam a condição de SAN das famílias e dos bairros ou comunidades, bem como sua inserção nas dinâmicas sistêmicas acima mencionadas. Como já explicado no marco analítico apresentado na seção anterior, chegou-se a três conjuntos de fatores principais que são: a) contexto biofísico; b) reprodução econômica das famílias e densidade sócio-organizacional; c) institucionalidade e acesso a bens e serviços públicos. 
Com base nesses três fatores, identificou-se entre um e quatro bairros urbanos e entre uma e quatro comunidades ou bairros rurais nos quais os fatores mencionados se manifestavam de forma distinta, no sentido de eles serem mais ou menos adversos em relação à SAN das famílias residentes. A escolha das famílias valeu-se também de informações obtidas junto às lideranças e outros agentes que atuam nos bairros-comunidades, visando contemplar situações diversas quanto ao perfil socioeconômico, participação em redes sociais e outros espaços e tempo de residência nos municípios. Foi analisada a consistência dos resultados obtidos com a utilização destes distintos instrumentos de pesquisa 23.

\section{CONCLUSÕES}

Este artigo apresentou uma matriz de análise de Sistemas Locais de Segurança Alimentar e Nutricional (Quadro 1). Os principais desafios para sua operacionalização residem no fato de seu escopo bastante amplo, que envolve diversos setores e instituições em diferentes campos, desde a produção até o consumo de alimentos. Partimos do pressuposto que analisar um Sistema, diferente de analisar uma política ou programas e ações específicas, implica abarcar as múltiplas questões que estão postas para a garantia de seus objetivos e princípios. No caso da SAN, um campo amplo, consequentemente complexifica o espectro de investigação e dificulta a profundidade da análise de todos os seus componentes. Dessa forma, tem-se uma análise geral de sua dinâmica, sem que seja possível aprofundar nenhum aspecto isoladamente.

Outro desafio reside na operacionalização de princípios tão complexos como intersetorialidade, equidade, descentralização, dentre outros, num mesmo estudo, o que remete às mesmas questões desafiadoras anteriormente ressaltadas.

Por fim, uma questão que deve ser destacada a partir da proposta aqui apresentada é que não há como analisar um sistema político institucional numa perspectiva normativa, ou seja, apenas avaliar seu desempenho no que se refere ao alcance de seus objetivos, princípios e diretrizes, comparando sua dinâmica "real" com um formato "ideal" previsto nas bases legais. Existe sempre um sistema real de fluxos, relações sociais e políticas que, no caso da SAN, define e condiciona de que forma os alimentos são produzidos, comercializados, acessados e suas consequências sobre o perfil de consumo e a situação geral de SAN da população.

Portanto, as interelações entre as potencialidades e desafios locais, no que se refere a existência de instituições, programas, atores e ações voltadas para garantir diferentes aspectos da SAN e os processos econômicos, políticos e sociais que definem o Sistema Alimentar existentes devem ser analisadas.

Um Sistema político institucional não gera processos e práticas iníquas de forma indissociada dos valores, processos e dinâmicas sociais que condicionam a distribuição dos diferentes tipos de 
recursos existentes em cada contexto local (terra, bens, recursos naturais, financeiros, políticos, dentre outros). Ou seja, os dois sistemas (alimentar e político institucional) estão profundamente interligados. Interesses e redes políticas atravessam agentes governamentais, agentes econômicos que produzem e comercializam os alimentos e afetam todas as dinâmicas e processos em curso. A interação entre esses fluxos econômicos e políticos, dentre os quais se incluem os próprios processos de implementação dos programas, é que "produz" em cada contexto local dinâmicas mais ou menos equitativas, que contribuem para fortalecer ou por vezes fragilizar a noção de direitos e uma articulação virtuosa entre os atores, no sentido da intersetorialidade, de modo a qualificar as políticas públicas.

\section{REFERÊNCIAS BIBLIOGRÁFICAS}

1. Anjos, L. A., Burlandy, L Construção do conhecimento e formulação de políticas públicas no Brasil na área de Segurança Alimentar e Nutricional. C S Col 2010; 15 (1): 19-30.

2. Brasil. Presidência da República. Casa Civil. Lei Nº 11.346 de 15 de setembro de 2006. Cria o Sistema Nacional de Segurança Alimentar e Nutricional - SISAN com vista a assegurar o direito humano á alimentação adequada e dá outras providências. Brasília: Presidência da República, 2006.

3. Pinheiro, A.R. O. Análise histórica do processo de formulação da política nacional de segurança alimentar e nutricional (2003-2006): atores, idéias, interesses e instituições na construção do consenso político. Tese defendida no Programa de Pós- graduação em política social. Departamento de Serviço Social da Universidade de Brasília, como requisito parcial para obtenção do Título de Doutor em política social; 2009.

4 . Pessanha, L. Santos, C.V. Mitchell, P.V. Indicadores para avaliar a Segurança Alimentar e Nutricional e a garantia do Direito Humano à Alimentação: metodologia e fontes de dados. Trabalho apresentado no XVI Encontro Nacional de Estudos populacionais. Caxambu, 2008. Disponível em www.abep.nepo.unicamp.br/encontro2008/.../ABEP2008_1489.pdf

5 . Santos, S, M.C. e Santos, L.M.P. Avaliação de políticas públicas de segurança alimentar e combate à fome no período de 1995-2002. Abordagem metodológica. Cad. Saúde Pública, 23 (5):1029-1040, 2007. Disponível em: http://www.scielo.br/pdf/csp/v23n5/05.pdf

6. Correa, A.M.S. Leon, L.M; Sampaio, M.F.A. Panigassi, G; Escamilla, R.P. Insegurança Alimentar no Brasil; do desenvolvimento de instrumentos de medida aos primeiros resultados nacionais. IN Paes-Sousa, R \& Vaitsman, J. Avaliação de políticas e programas do MDSResultados. Vol 1 Segurança Alimentar e Nutricional. Brasília: SAGI/MDS, 2007. 
7 . Burlandy, L; Magalhães, R \& Maluf, R (coords). Construção e promoção de sistemas locais de segurança alimentar e nutricional: aspectos produtivos, de consumo, nutricional e de políticas públicas. Série Relatórios Técnicos 3. Rio de Janeiro: CPDA/UFRRJ, 2006. Disponível em http://www.ufrrj.br/cpda/ceresan/docs/relatoriotecnico3.pdf.

8. Hartz Z.M.A., organizador. Avaliação em saúde: dos modelos conceituais à prática na análise da implantação de programas. Rio de Janeiro: Editora Fiocruz; 2000. p. 49-88.

9. Viacava, F. et al. Uma metodologia de avaliação do desempenho do sistema de saúde brasileiro. Ciênc. saúde coletiva, Set 2004, vol.9, no.3, p.711-724. Disponível em http://www. scielosp.org/pdf/csc/v9n3/a16v09n3.pdf.

10 . Viacava, F. et al. Uma metodologia de avaliação do desempenho do sistema de saúde brasileiro. Ciênc. saúde coletiva, Set 2004, vol.9, no.3, p.711-724. Disponível em http://www. scielosp.org/pdf/csc/v9n3/a16v09n3.pdf.

11. Vasconcellos, M. J. E. Pensamento sistêmico - o novo paradigma da ciência. Campinas, Papirus, 2003.

12. Capra, F. A teia da vida, uma nova compreensão científica dos sistemas vivos. São Paulo: Editora Cultrix, 2003.

13. Santos, B. S. Crítica à Razão Indolente: contra o desperdício da experiência. S. Paulo: Cortez, 2001.

14. Hirschman, A. O. Desenvolvimento por efeitos em cadeia: uma abordagem generalizada. Estudos CEBRAP, N. 18, Outubro-Dezembro/1976.

15. Maluf, R. S. Atribuindo sentido(s) ao desenvolvimento econômico. Estudos Sociedade e Agricultura, R. Janeiro, n. 15, 2000, p. 53-86.

16. Sen, A. The Concept of Development. In: Chenery, H. and Srinivasan, T.N. (eds.). Handbook of Development - Vol. I. Amsterdan, North Holland, 9-26, 1988.

17. Santoro, P. e Pinheiro, E. (orgs). O município e o solo rural. São Paulo: Instituto Polis, 2004.

18. Barraclough, S. L. And end to hunger? The social origins of food strategies. London, Zed Books, 1991. 
19. Hirschman, A. O. Rival views of market society and other recent essays. N. York, Viking Press, 1986.

20 . Flick, Uwe. Desenho da pesquisa qualitativa. Porto Alegre: Artmed, 2009.

21. Minayo MCS, Assis SG, Souza ER, organizadoras. Avaliação por triangulação de métodos: abordagem de programas sociais. Rio de Janeiro: Editora Fiocruz; 2005. 244 pp.

Artigo apresentado em 07-12-14

Artigo aprovado em 16-02-15

Artigo publicado no sistema em 22-12-15 